\title{
Matched Filter Based Spectrum Sensing Technique for 4G Cellular Network
}

\author{
SHADAB KALHORO*, FAHIM AZIZ UMRANI*, MUSTAHSAN ALI KHANZADA*, AND \\ LIAQUAT ALI RAHOO*
}

RECEIVED ON 18.10.2017 ACCEPTED ON 19.02.2019

\begin{abstract}
Modern and fast developments of wireless technologies have directed to a great demand for resources. It can be seen in the study that the range of existing spectrum is not used effectively, therefore the frequency band should be observed to ensure proper usage and to have the information of primary or licensed user is very much essential. In this research work uplink of LTE (Long-Term Evolution) is observed through MF (Matched Filter) spectrum sensing technique of CR (Cognitive Radio) network. This method examines the existence of signals in minimum possible time, reduces the hindrances between secondary users, increases accurateness of sensing and provides finest choice of threshold. In Uplink the System model which is used is known as SC-FDMA (Single Carrier Frequency Division Multiple Access). Entire simulation/results are prepared in MATLAB environment. This study also provides graphical contrast of simulated and theoretical results of matched filter and energy detection technique.
\end{abstract}

Key Words: Cognitive Radio, Matched Filter, Spectrum Sensing Techniques, Single Carrier Frequency Division Multpile Access Technique.

\section{INTRODUCTION}

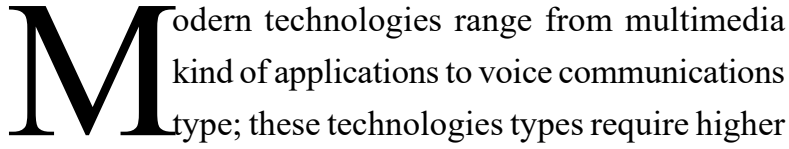
transmission data rates, however, the radio band has a number of constraints that it does not accommodate a growing amount of higher rates of data. Power and bandwidth/spectrum are not costly as well extremely inadequate. Licensing of spectrum range is precious technique that guarantees an inefficient use of the spectrum band. Given that demand in the market shows an unbalanced use and a shortage of spectrum, it is important to introduce new procedures or authorization rules which permit an efficient and dynamic method to be used with the existing band effectively[1].

FCC (Federal Communications Commission) presides above the utilization of the band with certain allocated ranges to the primary user. On the other side number of bandwidths is not utilized properly, whereas others are overfilled. In general user service quality is decreased by crowded band.

This result in a new structure is known as CR, which is commenced to accommodate to many consumers with high rates of data. This is regarded as the best way out

Authors E-Mail: (shadabkalhoro@hotmail.com, faheemaziz.umrani@faculty.muet.edu.pk, mustahsan.ali@isra.edu.pk, liaquatalirahoo2003@gmail.com)

* Institute of Information \& Communication Technologies, Mehran University of Engineering \& Technology, Jamshoro, Pakistan.

This is an open access article published by Mehran University Research Journal of Engineering and Technology, Jamshoro under the CC by 4.0 International License. 
for this type of problem. The intellect layer in sensing of spectrum is indicated in a CR that has the purpose of controlling spectrum frequency band. The sensing spectrum layer performs two key tasks; first find the band and decide which portion is empty (white spaces) also findout the occurrence of licensed consumer; second, the CR decides that the free spectrum will be assigned to unauthorized users without the intervention of licensed users [2].

LTE is required to be more flexible because of the utilization of the bandwidth and is expected to give high information rates and latencies at the edges of the cell. Therefore, the system of transmission for mobile station to base station (uplink) and base station to mobile station (downlink) is required to be equipped for new results superior to $3 \mathrm{G}$ networks [4]. Most current techniques adopt Sc-FDMA for transmission of the uplink. It can make use of multiplexing of orthogonal frequency, modulation of single carrier and equalization of frequency domain for the performance improvement of PAPR (Powered Air Purifying Respirator). But, SCFDMA experiences some challenges, the critical one of them is CFO (Chief Financial Officer) which directs to ICI (InterCarrier Interference) and MAI (Multiple Access Interference) [5].

In LTE system, for transmission over uplink technique SCFDMA has been selected, unlike OFDMA (Orthogonal Frequency Division Multiple Access) it changes signal time domain into frequency domain signal which can be used to improve throughput of system. Also mobile terminals can take advantage in terms of power transmit energy from feature known as low peak to average power [6].

\section{NON-COOPERATIVE DETECTION TECHNIQUES}

The recognition of primary user signal is essentially based on non-cooperative detection. The best known noncooperative detection techniques used for spectrum sensing are: energy detection, cyclo stationary, wavelength and matched filter [2].

Currently, the practice which is famous for detection has been an energy detection technique but the main problem of this procedure is that with a low SNR (Signal-to-Noise Ratio), this provides regrettable performance, while in noise licensed consumers have no adequate dissimilarity among them. While SNR is increased with the method of MF; it also requires a minimum time to obtain high amount of processing gain.

\section{MATCHED FILTER SPECTRUM SENSING}

A linear filter is also identified as MF which needs less time for detection and is considered as good noise detector. On behalf of a known input signal, the filter has been designed to extend the SNR output. For this reason, matching filter needs information about the shape of pulse, bandwidth and modulation and format of frame etc. [7]. The MF spectrum acquisition needs a priori PU (Primary User) information to accommodate a secondary consumer. In association with further detection practices, MF has the utmost SNR of the received signal.

Its process is identical as that of correlation; using filter, an unknown signal is convolved. Its desire reaction must be repeated and changed over time through the position signal.The Equation (1) of MF expressed in [8] is given by:

$$
\mathrm{y}[\mathrm{n}]=\sum_{\mathrm{n}=-\infty}^{\infty}(\mathrm{h}[\mathrm{n}-\mathrm{k}] \mathrm{x}[\mathrm{k}])
$$

At this point ' $h$ ' is the response of filter called as the impulse response; ' $\mathrm{x}$ ' is known as unknown signal and $\mathrm{y}[\mathrm{n}]$ represents the MF. The method of spectrum detection in the MF involves the demodulation of the received signal $\mathrm{y}[\mathrm{n}]$, therefore, it requires more information compared to other techniques. 
If the signal passes through the filter, it will make decision whether the signal is free of noise or not; filter will give emphasis to the signal $\mathrm{s}(\mathrm{t})$ and on the same time to minimizes the noise factor. Filter should be designed to peak out the signal components while at the same time suppressing the amplitude of the noise. It is considered a strong contrast between noise and signal; if there is a signal on the output side a high peak will appear; if there is no signal at any given time no peak would appear. This agreement would allow reaching at a decision that whether or not the signal is available with smallest amount of probability of error [9]. Increasing signal factors and reduction of the noise factors are the most important motive of this filter. At the output side this is probably the amplitude of the maximum signal with amplitude of the noise ratio at various points. This shows that square amplitude measurement is more appropriate [10].

\section{SYSTEM MODEL}

In the Uplink portion of LTE physical layer, a SC-FDMA is selected as fundamental scheme of multiple access. Many of the useful blocks of OFDMA are reused, so they are considered as a scheme of multicarrier. Fig. 1 shows this multiuser scenario with a block diagram of transceiver of SC-FDMA. In addition, compared to OFDMA, SCFDMA in the transmitter reduces energy consumption because it has a smaller PAR (Peak/Average Ratio). The advantage of this arrangement is elimination of intermodal interference (ISI). Different sub-carriers of orthogonal are used on the transmission side of the SC-FDMA to transmit data representations in succession [7].

\subsection{Transmitter}

Transmitter converts input binary data in to a set of adjusted subcarrier. The baseband called transmitter modulator restores the binary input data to the multilevel complex number " $\mathrm{X}_{\mathrm{N}}$ ". Consequently, transmitter converts serial modulated data in to the parallel data streams N; to carry out N-DFT modulation in subcarrier. In N-DFT point modulated data in time domain is converted in to frequency domain. Mapping is the next step in which symbol output of N-DFT is mapped to one of the M subcarrier orthogonal signals. Following this mapping IDFT (Inverse Discrete Fourier Transform) is implemented that changes complex symbols of frequency domain in to signals of time domain. Symbols are converted into serial from parallel. This conversion renovates output of time domain in an appropriate structure for the transmission of modulation and carrier radio frequency. Transmitter also performs processing of signal and offers guard time known as CP (Cyclic-Prefix) to prevent from IBI (Inter Block Interference). The reason of $\mathrm{CP}$ is to place number of symbols. For discrete time this linear convolution is converted in to circular convolution. Subsequently in frequency domain many sophisticated ways of

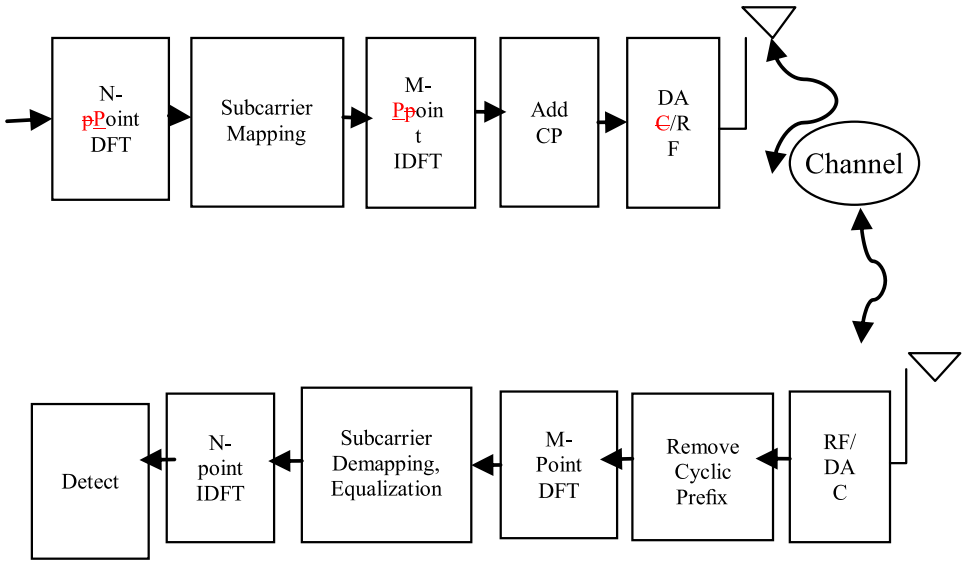

FIG. 1. TRANSCEIVER OF SC-FDMA [11]

Mehran University Research Journal of Engineering \& Technology, Volume 38, No. 4, October, 2019 [p-ISSN: 0254-7821, e-ISSN: 2413-7219] 
equalization can be performed to acquire relief from distortion of the medium.

\subsection{Channel}

To provide simulation in communication system, it is important to have an enhanced channel quality of a model for sending reliable data between transmitter and receiver. the requirements like nonstop propagation fading and multiple routes are important because that requires time variant medium that is difficult to be accurate as it occupies continuous assessment. Primarily fading is the consequence of multi path transmission. This leads to an amount of aspects as scattering, diffraction and reflection [12]. Multi path causes can be considered as constructive and destructive interference which lead to phase change and frequency change. It is possible to have direct connection with LOS (Line-of-Sight) but in reality it is not reliable because it is not measured as a good estimate.

\subsection{Rayleigh Distributions}

Rayleigh distribution is used to illustrate the effect of small scale fading. This provides the enhanced evaluation to decide the fading attributes amplitude of B-LOS (NonLine of Sight) surroundings for faded mobile medium. Adequacy of this model is based on the allocation of Rayleigh which can be seen in huge cities where many objects could diffract, refract and attenuate the signal. Path loss or shadowing could have dissimilar properties in the surroundings therefore fading is superimposed.

\subsection{Receiver}

Receiver receives a low-frequency signal during demodulation. Signal can be converted back in to a digital form by performing digital processing which eradicate the CP. Subsequently the elimination of $\mathrm{CP}$ information would be renewed in to parallel. After the conversion, the receiver changes the received frequency in the frequency domain using the DFT blocks; subcarriers are subsequently demapped[12]. Next to that, frequency domain equalization is performed. In addition,the compensation for the frequency ratio equalizer is estimated using the MMSE (Minimum Mean Square Error), which is the most commonly available equalizer [12]. The use of IDFT equalizer signal is renovated in reformatted time domain which is again renovated in data of serial mode, finally in time domain detection is performed.

\section{RESULTS AND DISCUSSION}

MATLAB 2015 has been used for simulation. Simulations are carried out by developing LTE system for Uplink for three users. For this work Table 1 provides the overview of parameters which are used in simulation. Over the Uplink of LTE Matched filter detection technique is compared with energy technique of detection; uplink uses SC-FDMA. Here to find out the different data rates of multiples user these two techniques are observed. Fig. 2 exposed the consequences of how the BER (Bit Error Rate) modifies against the $E_{b} / N_{o}$ (Bit Energy to Noise Ratio) values for scheme of modulation as BPSK (Binary Phase Shift Keying). Fig. 2 also expresses the comparison of Energy and MF detection performance. From the result it can easily be concluded that at definite value of $\mathrm{E}_{\mathrm{b}} / \mathrm{N}_{\mathrm{o}}$, MF BER is much lesser than that of energy detection. Also it could easily be observed that MF graph is much nearer to the theoretical line of BER. MF gives better performance when $E_{b} / N_{o}$ equals to $30 \mathrm{~dB}$ or less is practically reasonable. Fig. 3 expresses that all three users are concurrently present at the same band of frequency as their energy lies within $800 \mathrm{MHz}$. Receiver is intelligent enough to sense any desired user using the applied technique.

TABLE 1. SIMULATION PARAMETERS USED FOR LTE UPLINK

\begin{tabular}{|c|c|}
\hline System & LTE Uplink SC-FDMA \\
\hline Carrier Frequency & $800 \mathrm{MHZ}$ \\
\hline Subcarrier & 52 \\
\hline Length of FFT & 64 \\
\hline Channel Type & Rayleigh Channel \\
\hline Range of Eb/No in dB & $0-30$ \\
\hline Modulation Scheme & BPSK \\
\hline Numberof Users & Three \\
\hline
\end{tabular}

Mehran University Research Journal of Engineering \& Technology, Volume 38, No. 4, October, 2019 [p-ISSN: 0254-7821, e-ISSN: 2413-7219] 


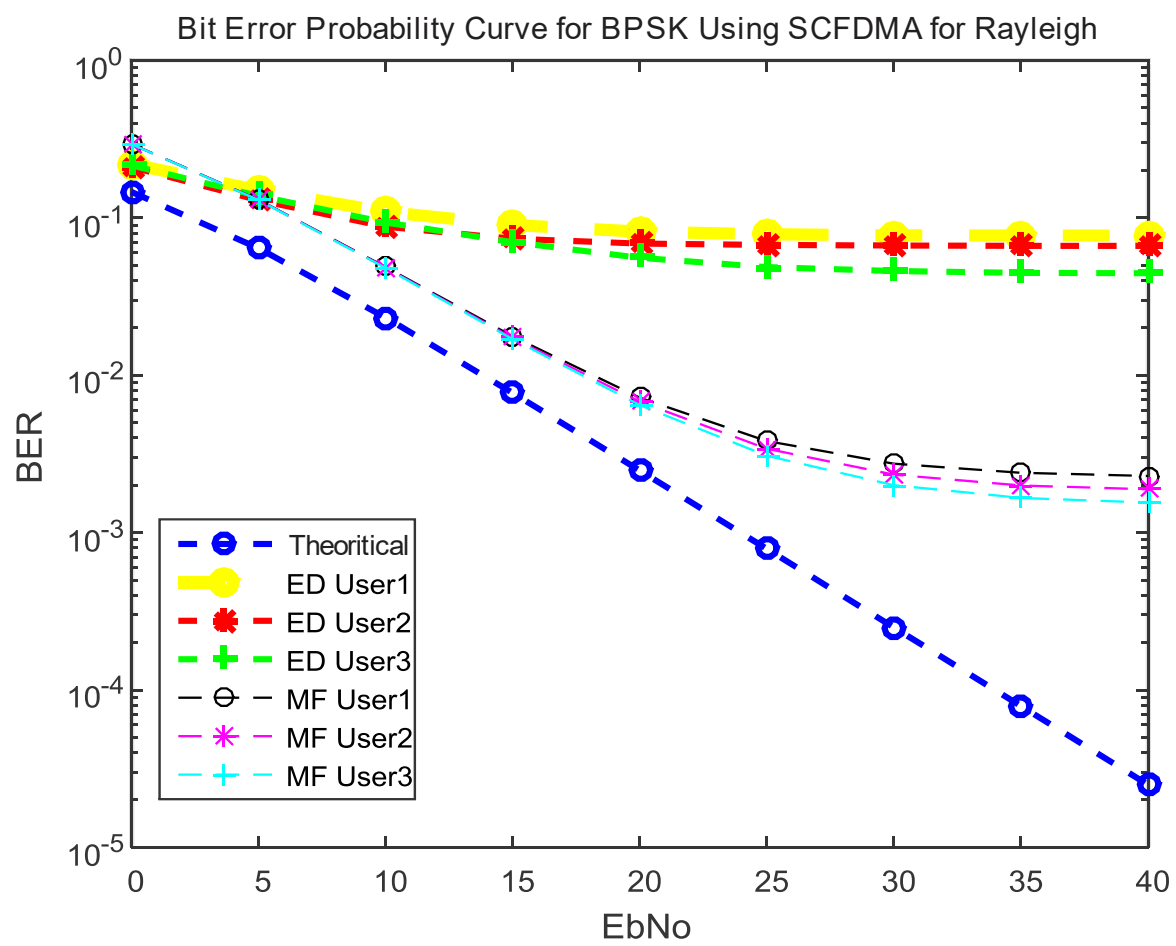

FIG. 2. BER VS. EB/NO FOR 800 MHZ WITH 52 SUBCARRIERS

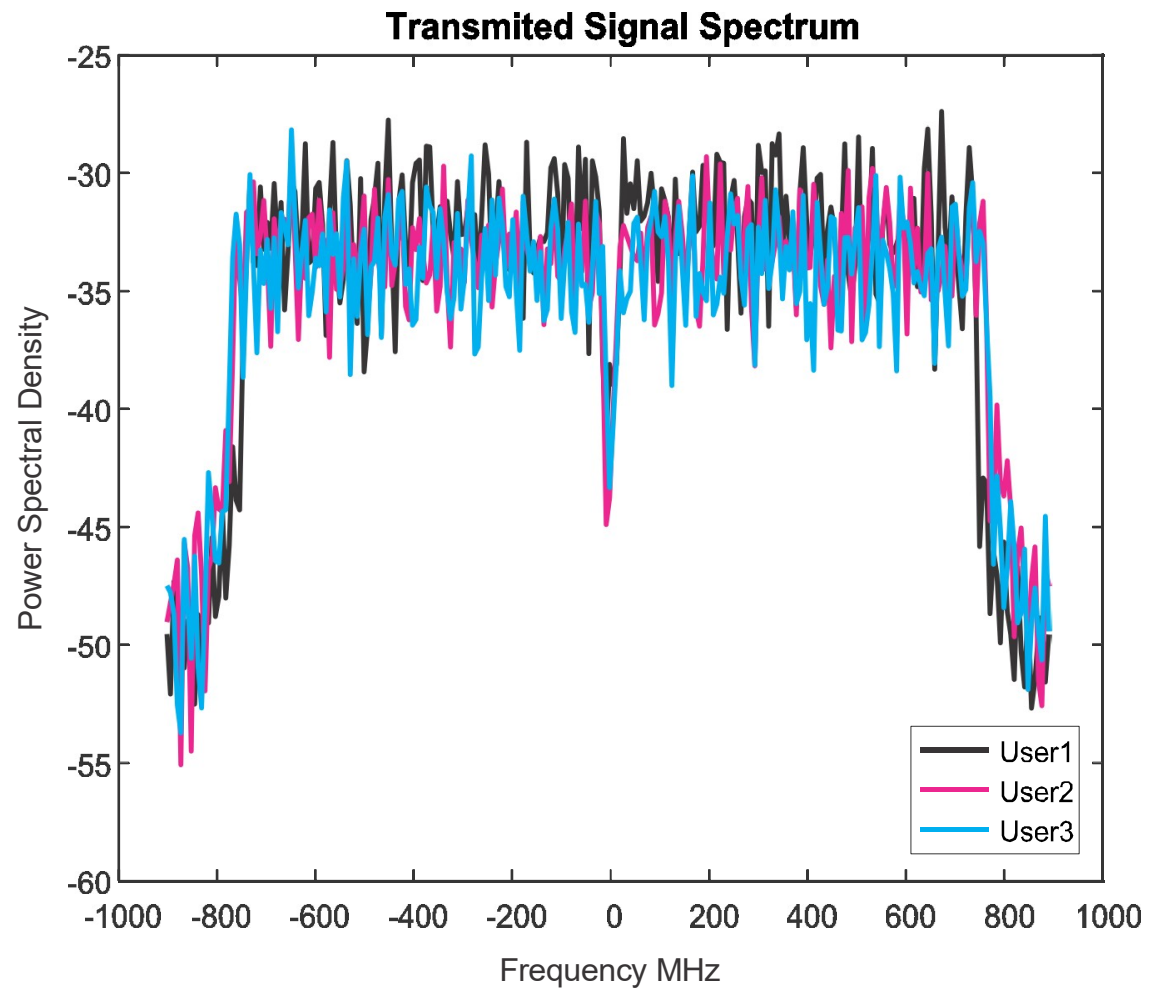

FIG. 3. POWER SPECTRAL DENSITY FOR ALL THREE USERS 


\section{CONCLUSION}

Paper focuses on comparison of two techniques MF and Energy detection technique for the transmission of multiple users on uplink of LTE using modulation scheme BPSK. From the outcomes it could be concluded that as compared to Energy detection technique MF gives lesser $\mathrm{BER}$ at specific value of $\mathrm{E}_{\mathrm{b}} / \mathrm{N}_{\mathrm{o}}$.

In particular, further research should be carriedout investigate Cyclostationary and other blind techniques of spectrum sensing for cellular systems $4 \mathrm{G}$ LTE and LTE-A.

\section{ACKNOWLEDGEMENT}

The authors are thankful to the Institute of Information \& Communication Technologies, Mehran University of Engineering \& Technology Jamshoro, Pakistan, for providing the required resource to perform this research.

\section{REFERENCES}

[1] Subhedar, M., and Birajdar, G., "Spectrum Sensing Techniques in Cognitive Radio Networks: ASurvey", International Journal of Next-Generation Networks, Volume 3, No, 2, pp. 37-51, 2011. Guo, X., and Song, P., "Simulink Based LTE System Simulator", Department of Signals and Systems Chalmers University of Technology Göteborg, Sweden, 2010.

[3] Uzairue, S.I., Nwukor, F., Alashiri, O., andAmiaze, P., "A Review: Evolution of 5G, Security and Multiple Access Schemes in Mobile Communication",International Research Journal of Engineering and Technology, Volume 4, No. 12, pp. 1277-1279, December, 2017.

[4] Al-Soufy, K.A., and Al-kamali, F.S., “An Efficient Transmission of Image Over SC-FDMA System in the Presence of CFO", Journal of Communications Technology, Electronics \& Computer Science, Volume 18, pp. 8-18, 2018.
[5] Vindhya, J., "Sensing of Spectrum for SC-FDMA Signals in Cognitive Radio Networks", International Research Journal of Engineering \& Technology,[e-ISSN: 23950056], Volume4, No. 9,September, 2017.

[6] Verma, D., and Gupta, A., "QoS and Energy Efficient Resource Allocation in Uplink SC-FDMA Systems-A Review", International Journal Engineering Development and Research, Volume 5, No. 4, [ISSN: 2321-9939], 2017.

[7] Verma, P.K., Taluja, S., and Dua, R.L., "Performance Analysis of Energy Detection, Matched Filter Detection \&Cyclostationary Feature Detection Spectrum Sensing Techniques", Indian Streams Research Journal, Volume 2, No, IX, 2012.

[8] Shukla, R., and Sharma, D., "Algorithm for Detection of Primary Users in Cognitive Radio by Spectrum Sensing Using PSD", International Journal of Research in Advent Technology, Volume 1, No. 5. 2013.

[9] Vadivelu, R., Sankaranarayanan, K., and Vijayakumari, V., "Matched Filter Based Spectrum Sensing for Cognitive Radio at Low Signal to Noise Ratio", Journal of Theoretical \& Applied Information Technology, Volume 62, No. 1, 2014.

[10] Eftekhari, A., Romberg, J., and Wakin, M.B., "Matched Filtering from Limited Frequency Samples", IEEE Transactions on Information Theory, Volume 59, No. 6, pp.3475-3496, 2013.

[11] Kalwar, S., Umrani, F.A., and Magarini, M., "Analysis of Effect of Carrier Frequency Offset on Performance of LTE Uplink", Proceedings of IEEE $3^{\text {rd }}$ International Conference on Digital Information, Networking, and Wireless Communications, pp. 35-38, 2015.

[12] Suarez, M., and Zlydareva, O., "LTE Transceiver Performance Analysis in Uplink Under Various Environmental Conditions", Proceedings of IEEE $4^{\text {th }}$ International Congress on Ultra-Modern Telecommunications and Control Systems and Workshops, pp. 84-88, 2012. 